\title{
Through the AGB Towards a Planetary: A Hydrodynamical Simulation
}

\author{
M. Steffen, D. Schönberner, K. Kifonidis and J. Stahlberg
}

Astrophysikalisches Institut Potsdam, Potsdam, Germany

Based on the mass-loss description developed by Blöcker (1995, A\&A, 297, 727), we present first exploratory computations of the dynamical evolution of a dusty stellar wind envelope around an intermediate mass star during the last 300000 years on the AGB and its transformation into a planetary nebula during the following 5000 years of post-AGB evolution. To model the dynamics of the cool dusty envelope, we used a two-component (gas/dust) 1D radiation hydrodynamics code which computes the radiation pressure on dust grains and the structure of the envelope in a self-consistent way, including the variable frictional coupling between dust and gas. The grains are either carbon or oxygen based and of single size and spherical shape.

We find that the pronounced variations of the mass loss rate during the so called "thermal pulses" lead to drastic changes of the emergent spectral energy distributions, even on rather short time scales. As a consequence, the modeled objects on the upper AGB trace extended loops in the IRAS two-color-diagram. The existence of AGB stars with detached shells is a natural consequence of the sharp decrease of mass loss following a thermal pulse. At the end of the AGB evolution the density and velocity structure of the wind envelope reflects the previous mass-loss history and differs considerably from that of stationary outflows. No significant differences between carbon and oxygen-rich envelopes are found.

The post-AGB phase has been modeled with a newly developed code that computes the radiation hydrodynamics of interacting winds with fully time-dependent ionization, heating and cooling processes, using our final AGB wind envelope as an initial structure. The development of observationally well-known structures of planetaries are found to be the natural consequences of the temporal changes of the surface and wind parameters of the central star, which has a final mass of $0.6 \mathrm{M}_{\odot}$ after the heavy mass loss ceases. We show that the often found double-shell structures are entirely formed by hydrodynamical effects and have nothing to do with the mass-loss history along the upper AGB, contrary what is often stated in the literature. Another important conclusion is that, for double-shell planetaries, the Doppler splitting of the main components of prominent emission lines like O III, $\lambda 5008 \AA$, does not give an appropriate estimate of the true expansion velocity of the nebula. Rather, the velocity measured from the faint wings (shoulders) of the line profiles is a better indicator of the true expansion rate and may be used to estimate kinematical ages of planetaries. One should be aware, however, that the outer rim of a planetary is defined by a shock front, the temporal displacement of which is not given by a material velocity and hence is not accessible to spectroscopic measurements. 